Systematic Review

\title{
Perineural Dexamethasone Added to Local Anesthesia for Brachial Plexus Block Improves Pain but Delays Block Onset and Motor Blockade Recovery
}

Nebojsa Nick Knezevic, MD, PhD ${ }^{1,2}$, Utchariya Anantamongkol, PhD, MD, and Kenneth D. Candido, MD ${ }^{1,2}$

From: ${ }^{2}$ Department of Anesthesiology, Advocate Illinois Masonic Medical Center, Chicago, IL; 2Department of Anesthesiology, University of Illinois, Chicago, IL

Dr. Knezevic is Director of Anesthesiology Research and Clinical Assistant Professor, Department of Anesthesiology, Advocate Illinois Masonic Medical

Center, Chicago, IL; Department of Anesthesiology, University of Illinois, Chicago, IL. Dr.

Anantamongkol is a Resident, Department of Anesthesiology, Advocate Illinois Masonic Medical Center, Chicago, IL. Dr. Candido is Professor and Chairman,

Department of Anesthesiology, Advocate Illinois Masonic Medical Center, Chicago, IL; Department of Anesthesiology, University of Illinois, Chicago, IL.

Address Correspondence: Kenneth D. Candido, M.D. Department of Anesthesiology, Advocate Illinois Masonic Medical Center, Chicago, IL

$836 \mathrm{~W}$. Wellington Ave. Suite 4815 Chicago, IL 60657

E-mail: kdcandido@yahoo.com

Disclaimer: There was no external funding in the preparation of this manuscript.

Conflict of interest: Each author certifies that he or she, or a member of his or her immediate family, has no commercial association (i.e., consultancies, stock ownership, equity interest, patent/licensing arrangements, etc.) that might pose a conflict of

interest in connection with the submitted manuscript.

Manuscript received: 06-24-2014 Revised manuscript received: o8-12-2014 Accepted for publication: 08-20-2014

Free full manuscript: www.pain physicianjournal.com
Background: Multiple studies have shown that perineural dexamethasone improves postoperative analgesia. However, some studies have shown minimal benefit, and have raised concerns regarding adverse physio-chemical effects of perineural dexamethasone. Furthermore, there is a paucity of studies wherein control (IV) dexamethasone was considered.

Objective: The purpose of this meta-analysis was to evaluate the effectiveness of different concentrations of perineural dexamethasone injection on postoperative analgesia, as well as complications from its use for brachial plexus blocks.

Methods: A systematic literature search was conducted using the Cochrane Central Registry of Controlled Trials, PubMed, and Scopus. Trials comparing control and local dexamethasonetreated groups, and those which reported duration of analgesia and/or pain scores/opioid consumptions were selected. Meta-analysis was performed using the Review Manager (RevMan) software 5.1.

Results: Fourteen studies consisting of a total of 1,022 patients were included. Perineural dexamethasone significantly prolonged the duration of postoperative analgesia in patients receiving both low-dose $(4-5 \mathrm{mg})$ [SMD $2.41(95 \% \mathrm{Cl}: 1.47,3.35 P=0<0.00001) \mathrm{I}^{2}=82 \%$ ], and higher-doses $(8-10 \mathrm{mg})$ [SMD $\left.4.46(95 \% \mathrm{Cl} 3.54,5.38 P<0.00001) \mathrm{I}^{2}=94 \%\right]$. However, the duration of motor block was also prolonged [SMD $2.52(95 \% \mathrm{Cl}: 1.06,3.98 P=0.0007) \mathrm{I}^{2}$ $=97 \%$ ] and dexamethasone delayed latency of onset of sensory [SMD $-0.49(95 \% \mathrm{Cl}:-0.89$, $-0.09 P=0.02) \mathrm{I}^{2}=76 \%$ ] and motor [SMD $-0.56(95 \% \mathrm{Cl}:-1.13,0.00 P=0.05) \mathrm{I}^{2}=87 \%$ ] blocks. Postoperative pain scores were improved at both 24 hours [SMD $-1.46(95 \% \mathrm{Cl}:-2.43$, $-0.50 P=0.003) \mathrm{I}^{2}=95 \%$ ] and 48 hours [SMD $-1.20(95 \% \mathrm{Cl}:-2.26,-0.13 P=0.03) \mathrm{I}^{2}=95 \%$ ] in dexamethasone-treated groups, whereas opioid consumption was reduced only at 48 hours [SMD $-\left.2.97(95 \% \mathrm{Cl}:-4.17,-1.76 P<0.00001)\right|^{2}=88 \%$ ]. Complications were comparable between control and dexamethasone-adjuvant groups, except for the excessively prolonged nerve block that was observed predominantly in the dexamethasone-adjuvant group.

Limitations: The limitations include different definitions used for the measurements of certain parameters such as the duration of analgesia and duration of motor block, number of studies assessing certain parameters having high heterogeneity, and varying types of local anesthetics used in various studies.

Conclusions: Perineural dexamethasone addition to local anesthetic solutions significantly improved postoperative pain in brachial plexus block without increasing complications. However, perineural adjuvant dexamethasone delayed the onset of sensory and motor block, and prolonged the duration of motor block. Smaller doses of dexamethasone $(4-5 \mathrm{mg})$ were as effective as higher doses (8 - $10 \mathrm{mg}$ ).

Key words: Dexamethasone, perineural, brachial plexus block, postoperative pain, metaanalysis, systematic review

Pain Physician 2015; 18:1-14 
$\mathrm{R}$ egional anesthesia is an excellent adjunct or alternative to general anesthesia for extremity surgery. It provides superior postoperative analgesia and hastens recovery from anesthesia. Although regional anesthesia has an opioid-sparing effect, occasionally postoperative analgesic duration is not adequate to compensate for the acute nociception associated with surgery in the early perioperative phase of healing. Many adjuvants including clonidine, neostigmine, epinephrine, tramadol, buprenorphine, and dexamethasone have been added to local anesthetics (LA) in an effort to enhance postoperative analgesia $(1,2)$. There remains controversy regarding the relative efficacy of these respective additives in that it remains unknown whether or not their effectiveness is due to a local, perineural, or a systemic effect from the absorbed agent.

Corticosteroids are widely used in peripheral nerve blocks for acute pain control and are routinely injected into the epidural space for treating radicular pain with a reliably acceptable side effect profile $(3,4)$. Intravenous dexamethasone has been previously shown to be opioid-sparing in the early postoperative phase between 24 - 48 hours following its administration $(5,6)$ and also serves to reduce postoperative nausea and vomiting (PONV) (7). Several clinical studies have evaluated the effectiveness of dexamethasone applied perineurally with LA in regional nerve blocks including epidural $(8,9)$ brachial plexus (10-23), femoral and sciatic (24), and facial and dental blocks $(25,26)$.

Surrogates used for measuring postoperative pain (e.g., duration of analgesia, pain scores, opioid consumption) appear to be improved by the addition of dexamethasone to LA agents injected perineurally $(6,7)$. However, some studies on peripheral nerve block have not identified significant effects of perineural dexamethasone on the postoperative duration of analgesia (27), pain scores at 24 hours $(24,27)$ and 48 hours $(16,19)$, opioid consumption $(15,16,18)$, as well as on reducing the latency of onset of the sensory or motor block $(14,18)$. Due to a concern of adverse physiochemical effects from perineural adjuvant dexamethasone, certain authors have recommended against its use as an adjuvant to LA or have suggested that alternative routes of administration (IV) are preferable $(11,28)$. Also, as several studies have used varying concentrations of dexamethasone ranging from $4 \mathrm{mg}$ to $10 \mathrm{mg}$ added to LA, an optimum dosing regimen remains undefined.

The aim of this meta-analysis was to evaluate the analgesic effects of adjunctive perineural dexametha- sone added to LA injections for brachial plexus blocks used to provide postoperative analgesia. The brachial plexus model was selected for evaluation because of the ample number of clinical trials wherein local dexamethasone application was studied. As low-dose perineural adjuvant dexamethasone has been previously recommended (28), we analyzed the effectiveness of varying doses on the duration of analgesia. We also gathered information regarding the duration of motor block, onset of blocks, and pain scores. Complications associated with perineural adjuvant dexamethasone use were also determined.

\section{Methods}

\section{Study Design}

The standards set by the Preferred Reporting Items for Systemic Reviews and Meta-Analyses (PRISMA) guidelines were used to construct this review (29).

\section{Eligibility Criteria}

In this meta-analysis we included studies that assessed the effects of perineural dexamethasone added to LA for brachial plexus blocks. The comparison was made between placebo/other adjunctive analgesics and dexamethasone-treated groups. Studies that met the following criteria were included: (1) human studies that included adult men and women ( $>18$ years) undergoing shoulder/upper limb surgeries; (2) prospective, randomized, double-blinded controlled trials published in English; (3) reported outcomes including either duration of analgesia or a numeric pain scale rating or postoperative opioid consumption. An effect from IV dexamethasone was not included in this analysis. Studies using dexamethasone with spinal or LA used for lower limb surgery or head and neck surgery, as well as dexamethasone used neuraxially for managing chronic radicular pain, were excluded. Trials recording either pain scores or opioid consumption, without a minimum 24 hours follow-up were also excluded.

\section{Literature Search}

A comprehensive literature search employing Medline (PubMed), Cochrane Central Register of Controlled Trials (CENTRAL), and Scopus database was undertaken. Search keywords included "dexamethasone," "steroids," "pain," "analgesia," "postoperative," "regional anesthesia," "regional analgesia," and "surgery," initially without language and species restrictions. Additional articles were found from references of selected studies. 
The search criteria were developed by all authors and independently conducted by 2 reviewers (UA and NNK). Relevant studies were carefully selected. Differences in literature inclusion/exclusion were decided by consensus.

\section{Outcome Parameters}

Duration of postoperative analgesia was the primary outcome. It was defined as "the interval between completion of dexamethasone adjuvant injection and time of first analgesic request" (11) or "the time when the patient first reported pain at the surgical site" $(10,12,16-20,22-23)$ or "the time when pain scores reached 8 on $0-10$ numeric rating scale (NRS)" $(14,21)$. However, one study measured the duration between the time of discharge from the postanesthesia care unit and the first report of pain at the incision site (15). Secondary outcomes were duration of motor block (from dexamethasone adjuvant injection until ability to move/raise hand or abduct the shoulder); onset of sensory and motor block (time from dexamethasone adjuvant injection until analgesia to pinprick and inability to move the hand or shoulder, respectively); NRS $(0-10)$ with movement (cough/sit) at 24 and 48 hours postoperatively; cumulative opioid consumption (oral morphine equivalents) at 24 and 48 hours postoperatively; and complications including nausea, hoarseness, dyspnea, and Horner's syndrome.

\section{Data Extraction}

Data were extracted independently by 2 authors (UA, NNK). Any disagreements were resolved by a discussion between the 3 reviewers (UA, NNK, KDC) for final agreement. The following data were listed in the data collection form (Tables 1,2): authors, year of publication, location of surgery, type of nerve block, number of total and treated patients, doses of dexamethasone (mg), LA and adjuncts used, eli-

Table 1. Characteristics of included studies in analysis.

\begin{tabular}{|c|c|c|c|c|c|c|c|c|}
\hline $\begin{array}{l}\text { Author/ } \\
\text { Year }\end{array}$ & $\begin{array}{c}\text { Number of } \\
\text { Treatment/ } \\
\text { Control }\end{array}$ & Procedure & $\begin{array}{c}\text { Type of } \\
\text { Brachial Block }\end{array}$ & $\begin{array}{l}{ }^{*} \text { Guidance } \\
\text { Technique }\end{array}$ & $\begin{array}{c}\text { Local } \\
\text { Anesthetics/ } \\
\text { Adjuncts }\end{array}$ & $\begin{array}{l}\text { Dexamethasone } \\
(\mathrm{mg})\end{array}$ & $\begin{array}{l}\text { Postoperative } \\
\text { Analgesia }\end{array}$ & $\begin{array}{l}\text { Modified } \\
\text { Jadad } \\
\text { Score }^{*}\end{array}$ \\
\hline $\begin{array}{l}\text { Persec et al, } \\
2014(10)\end{array}$ & $35 / 35$ & $\begin{array}{l}\text { Upper } \\
\text { extremity }\end{array}$ & Supraclavicular & US & $\begin{array}{c}0.5 \% \\
\text { levobupivacaine }\end{array}$ & 4 & Diclofenac IV PRN & 8 \\
\hline $\begin{array}{l}\text { Desmet } \\
\text { et al, } 2013 \\
\text { (11) }\end{array}$ & $49 / 46$ & Shoulder & Interscalene & NS & $0.5 \%$ ropivacaine & 10 & $\begin{array}{c}\text { Diclofenac } 50 \text { mg IV PRN } \\
\text { Paracetamol } 1 \text { g IV PRN } \\
\text { Piritramide } 15-20 \text { mg IM } \\
\text { PRN }\end{array}$ & 8 \\
\hline $\begin{array}{l}\text { Biradar et } \\
\text { al, } 2013 \\
(12)\end{array}$ & $30 / 30$ & $\begin{array}{l}\text { Upper } \\
\text { extremity }\end{array}$ & Supraclavicular & NS & $\begin{array}{l}1.5 \% \text { lidocaine } \\
1: 200,000 \\
\text { epinephrine }\end{array}$ & 8 & $\begin{array}{c}\text { Diclofenac } 1.5 \mathrm{mg} / \mathrm{kg} \text { IM PRN } \\
\text { Morphine } 2 \mathrm{mg} \text { IV q } 10 \mathrm{~min} \\
\text { until pain score }<3\end{array}$ & 8 \\
\hline $\begin{array}{l}\text { Kim et al, } \\
2012(13)\end{array}$ & $20 / 20$ & Shoulder & Interscalene & US & $\begin{array}{c}0.5 \% \\
\text { levobupivacaine } \\
1: 400,000 \\
\text { epinephrine }\end{array}$ & 5 & $\begin{array}{l}\text { Ketorolac IV PRN } \\
\text { Opioids IM PRN }\end{array}$ & 7 \\
\hline $\begin{array}{l}\text { Pathak } \\
\text { et al, } \\
2012 \text { (14) }\end{array}$ & $25 / 25$ & $\begin{array}{l}\text { Upper } \\
\text { extremity }\end{array}$ & Supraclavicular & NS & $\begin{array}{c}0.5 \% \text { bupivacaine } \\
1.5 \% \text { lidocaine } \\
1: 200,000 \\
\text { epinephrine } \\
\end{array}$ & 8 & $\begin{array}{c}\text { Diclofenac } 1-1.5 \mathrm{mg} / \mathrm{kg} \mathrm{IM} \\
\text { PRN }\end{array}$ & 7 \\
\hline $\begin{array}{l}\text { Tandoc } \\
\text { et al, } \\
2011(15)\end{array}$ & $\begin{array}{l}28 / 28 \\
30 / 28\end{array}$ & Shoulder & Interscalene & NS & $0.5 \%$ bupivacaine & $\begin{array}{l}4 \\
8\end{array}$ & $\begin{array}{c}\text { Acetaminophen } 325 \mathrm{mg}+ \\
\text { Hycrocodone } 7.5 \mathrm{mg} \text { PO PRN }\end{array}$ & 8 \\
\hline $\begin{array}{l}\text { Cummings } \\
\text { et al, } \\
2011 \text { (16) }\end{array}$ & $\begin{array}{l}54 / 54 \\
54 / 56\end{array}$ & Shoulder & Interscalene & US & $\begin{array}{l}0.5 \% \text { bupivacaine } \\
0.5 \% \text { ropivacaine }\end{array}$ & 8 & $\begin{array}{l}\text { Acetaminphen } 325-650 \mathrm{mg} \\
+ \text { Oxycodone } 5-10 \mathrm{mg} \mathrm{PO} \\
\text { PRN q } 4 \mathrm{~h}\end{array}$ & 8 \\
\hline $\begin{array}{l}\text { Islam et al, } \\
2011 \text { (17) }\end{array}$ & $30 / 60$ & $\begin{array}{l}\text { Upper } \\
\text { extremity }\end{array}$ & Supraclavicular & N/A & $\begin{array}{l}0.5 \% \text { bupivacaine } \\
2 \% \text { lignocaine }\end{array}$ & 8 & Not specified & 4 \\
\hline $\begin{array}{l}\text { Parrington } \\
\text { et al, } \\
2010 \text { (18) }\end{array}$ & $24 / 21$ & $\begin{array}{l}\text { Upper } \\
\text { extremity }\end{array}$ & Supraclavicular & US & $1.5 \%$ mepivacaine & 8 & $\begin{array}{c}\text { Fentanyl } 25 \mu \mathrm{g} \text { PRN q } 5 \mathrm{~min} \\
\text { Acetaminophen } 300 \mathrm{mg}+ \\
\text { Codeine } 30 \mathrm{mg} \text { PO PRN } \\
\text { Acetaminophen } 325 \mathrm{mg}+ \\
\text { Oxycodone HCl } 5 \mathrm{mg} \text { PO } \\
\text { PRN }\end{array}$ & 8 \\
\hline $\begin{array}{l}\text { Vieira et al, } \\
2010(19)\end{array}$ & $44 / 44$ & Shoulder & Interscalene & US & $\begin{array}{l}0.5 \% \text { bupivacaine } \\
1: 200,000 \\
\text { epinephrine } \\
75 \mu \mathrm{g} \text { clonidine }\end{array}$ & 8 & $\begin{array}{l}\text { Hydrocodone/Oxycodone/ } \\
\text { Hydromorphone PO PRN }\end{array}$ & 7 \\
\hline
\end{tabular}


Table 1 (cont.). Characteristics of included studies in analysis.

\begin{tabular}{|c|c|c|c|c|c|c|c|c|}
\hline $\begin{array}{l}\text { Author/ } \\
\text { Year }\end{array}$ & $\begin{array}{l}\text { Number of } \\
\text { Treatment/ } \\
\text { Control }\end{array}$ & Procedure & $\begin{array}{c}\text { Type of } \\
\text { Brachial Block }\end{array}$ & $\begin{array}{l}{ }^{*} \text { Guidance } \\
\text { Technique }\end{array}$ & $\begin{array}{l}\text { Local } \\
\text { Anesthetics/ } \\
\text { Adjuncts }\end{array}$ & $\begin{array}{c}\text { Dexamethasone } \\
(\mathrm{mg})\end{array}$ & $\begin{array}{l}\text { Postoperative } \\
\text { Analgesia }\end{array}$ & $\begin{array}{c}\text { Modified } \\
\text { Jadad } \\
\text { Score }^{\star}\end{array}$ \\
\hline $\begin{array}{l}\text { Yadav et al, } \\
2008(20)\end{array}$ & $30 / 30$ & $\begin{array}{l}\text { Upper } \\
\text { extremity }\end{array}$ & Supraclavicular & NS & $\begin{array}{c}\text { 1.5\% lignocaine } \\
1: 200,000 \\
\text { epinephrine }\end{array}$ & 4 & $\begin{array}{l}\text { Diclofenac } 50 \mathrm{mg} \text { PO PRN } \\
\text { Diclofenac } 75 \mathrm{mg} \text { IM PRN }\end{array}$ & 7 \\
\hline $\begin{array}{l}\text { Shrestha } \\
\text { et al, } 2007 \\
\text { (21) }\end{array}$ & $30 / 30$ & $\begin{array}{l}\text { Upper } \\
\text { extremity }\end{array}$ & Supraclavicular & N/A & $0.5 \%$ bupivacaine & 8 & Analgesics & 7 \\
\hline $\begin{array}{l}\text { Movefegh } \\
\text { et al, } 2006 \\
\text { (22) }\end{array}$ & $30 / 30$ & $\begin{array}{c}\text { Upper } \\
\text { extremity }\end{array}$ & Axillary & NS & $1.5 \%$ lidocaine & 8 & Not specified & 7 \\
\hline $\begin{array}{l}\text { Shrestha } \\
\text { et al, } 2003 \\
\text { (23) }\end{array}$ & $20 / 20$ & $\begin{array}{c}\text { Upper } \\
\text { extremity }\end{array}$ & Supraclavicular & N/A & $\begin{array}{c}0.5 \% \text { bupivacaine } \\
2 \% \text { lidocaine } \\
1: 200,000 \\
\text { epinephrine }\end{array}$ & $4-8$ & Not specified & 3 \\
\hline
\end{tabular}

* US indicates Ultrasound-guided method, NS Nerve stimulator guided method, N/A Not mentioned in the study.

Table 2. Characteristics of included studies in analysis.

\begin{tabular}{|c|c|c|c|c|c|c|}
\hline Author/Year & $\begin{array}{c}\text { Duration of } \\
\text { Analgesia }\end{array}$ & $\begin{array}{l}\text { Duration of } \\
\text { Motor Block }\end{array}$ & $\begin{array}{c}\text { Onset of Sensory } \\
\text { Block }\end{array}$ & $\begin{array}{c}\text { Onset of } \\
\text { Motor Block }\end{array}$ & $\begin{array}{l}\text { Pain } \\
\text { Score }\end{array}$ & $\begin{array}{c}\text { Opioid } \\
\text { Consumption }\end{array}$ \\
\hline Persec et al, $2014(10)$ & * & & & & * & \\
\hline Desmet et al<2013 (11) & * & & & & & \\
\hline Biradar et al, 2013 (12) & * & * & * & * & & \\
\hline Kim et al, 2012 (13) & & & & & * & \\
\hline Pathak et al, 2012 (14) & * & * & * & * & & \\
\hline Tandoc et al, 2011 (15) & * & * & & & & * \\
\hline Cummings et al, 2011 (16) & * & & & & * & \\
\hline Islam et al, 2011 (17) & * & & * & * & & \\
\hline Parrington et al, 2010 (18) & * & & * & * & * & * \\
\hline Vieira et al, 2010 (19) & * & * & . & & * & * \\
\hline Yadav et al, 2008 (20) & * & & * & * & & \\
\hline Shrestha et al, 2007 (21) & * & & * & * & & \\
\hline Movefegh et al, 2006 (22) & * & * & * & * & & \\
\hline Shrestha et al, 2003 (23) & * & & * & & & \\
\hline
\end{tabular}

gible primary and secondary outcomes, and complications (nausea, hoarseness, dyspnea, and Horner's syndrome).

For data lacking full description, we tried to contact the authors of the original publications. If the authors did not respond, numerical data were extracted from figures in their respective publications. Dexamethasone doses were varied from $4 \mathrm{mg}$ to $10 \mathrm{mg}$. In the analysis of duration of analgesia, the studies were categorized into "low dose" (4 - 5 mg) and "high dose" (8-10 mg) of dexamethasone. These ranges were also similar to a dose-response stratification in the systematic review of Waldron et al (6). Values for duration of analgesia, duration of motor block, and onset of motor and sensory block were converted in units of minutes (min).
Pain scores reported on the 11-point NRS $(0-10)$ were included. Opioid consumption was converted to oral morphine equivalents $(\mathrm{mg})$ using the ratios from Korff et al (30). Studies where control acetaminophen or non-steroidal anti-inflammatory drugs (NSAIDs) were used as postoperative pain medication adjuvants were excluded. Complications from brachial blocks were reported as per incidence and were included. Data presented in reporting systems other than stated were excluded. Values presented as median and interquartile were converted to mean and SD by using a formula (31).

\section{Risk of Bias}

Two authors (UA, NNK) independently assessed the methodological validity using a modified Jadad scale 
range from 0 to 8 , low to high quality (32). The score of $4-8$ is considered high quality and $0-3$ is low quality (33). Any discrepancies were resolved with inclusion of a third reviewer (KDC). The minimum score of included studies was 3 and maximum was 8 .

\section{Meta-analyses}

Meta-analysis was performed using the Review Manager (RevMan) Software 5.1 (The Nordic Cochrane Centre, The Cochrane Collaboration, Copenhagen, Denmark, 2008). For continuous data, the studies were reported as the standardized mean differences (SMD) with $95 \%$ confidence intervals $(\mathrm{Cl})$. Dichotomous data were presented with risk differences (RD) and $95 \%$ $\mathrm{Cl}$. Data were plotted using forest plots to evaluate treatment effects. Publication bias was evaluated using funnel plots. Heterogeneity was interpreted through 12 statistic, in which the value over $30 \%$ was considered heterogeneity. Significant statistics were considered when the $95 \% \mathrm{Cl}$ did not include 0 . Due to different surgical procedures and anesthetics used, a random effect model was used to generalize our findings, as similarly shown in previous studies $(5,6,34)$.

Duration of analgesia was further proceeded to subgroup analysis comparing the effects of dexamethasone with different LA. Only subgroups using one LA without any adjuncts were included. Different brachial block approaches were also analyzed for duration of analgesia. Subgroup analysis in the other parameters was not evaluated due to the insufficient number of eligible studies.

\section{REsults}

\section{Results of the Search}

In our extensive database research, a total of 1,644 publications were identified. Of the 39 examined full-text publications, 14 studies were subsequently included in this meta-analysis (Fig. 1) providing a total of 1,022 patients, in which 533 patients received dexamethasone as an adjuvant to perineural brachial plexus LA injections. Twenty-five studies were excluded: 10 studies used spinal subarachnoid or regional blocks other than brachial plexus techniques (35-40); 3 studies used intravenous regional anesthesia (IVRA) (41-43); 3 trials were performed in healthy volunteers (44-46); 2 trials were performed in chronic pain disease states such as lumbosacral radicular type pain $(8,47)$; one study was retrospective (48); and one had neither duration of analgesia reported as minutes nor pain scores provided at 24/48 hours (49). Fig. 1 shows the PRISMA flow diagram representing the disposition of retrieved publications. The median number of patients receiving dexamethasone in the included studies was 30.

Fourteen of the included studies contained at least one group wherein dexamethasone was part of the brachial plexus LA injection, which consisted of either dexamethasone added alone $(10,11,13,15,16,18,21,22)$ or in conjunction with epinephrine $(12,14,19,20,23)$ and with clonidine (19). Study groups were divided according to the added dexamethasone doses; 4 stud-

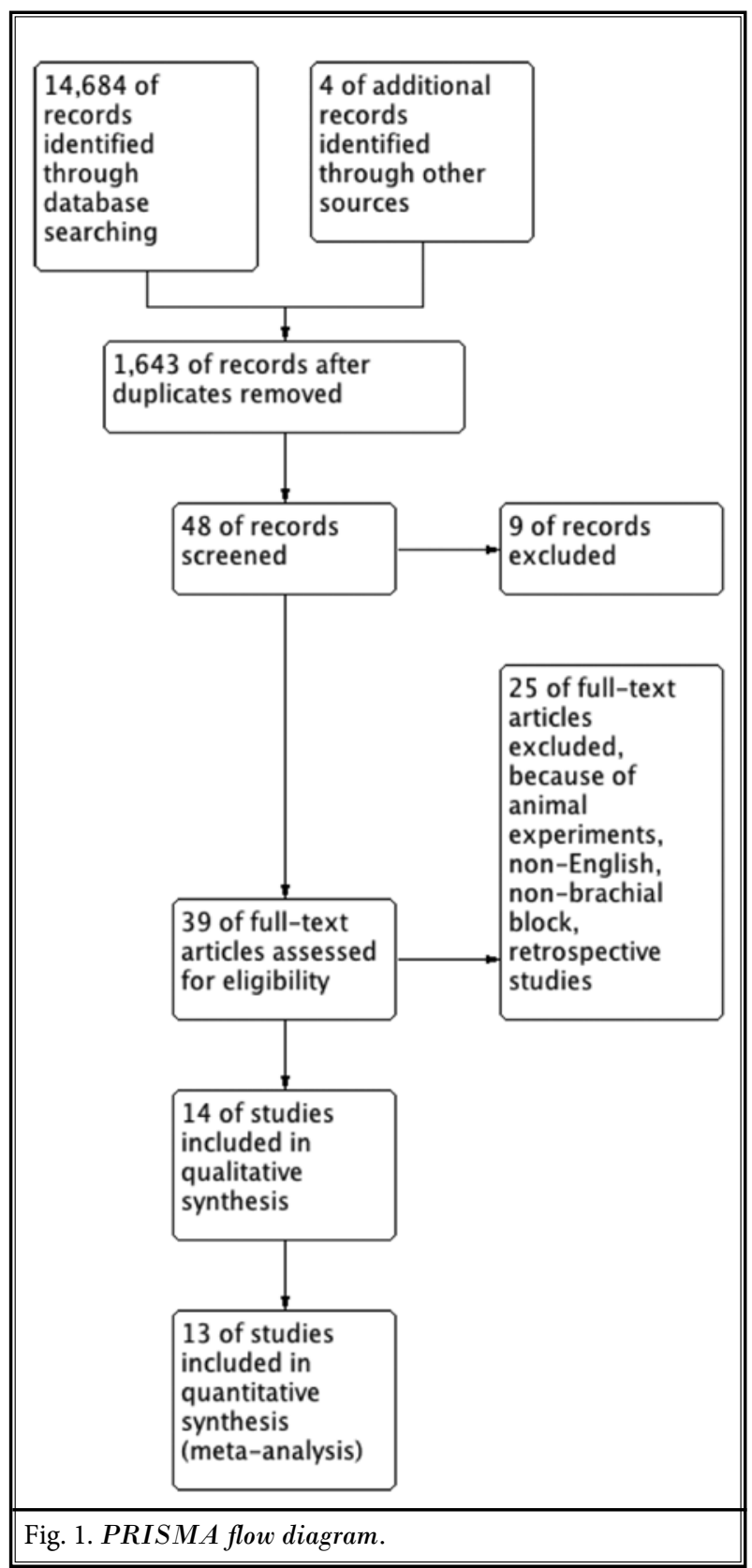


ies that used low doses of dexamethasone $(4-5 \mathrm{mg}$ ) $(10,13,15,20), 10$ studies that used high doses of dexamethasone (8 - $10 \mathrm{mg})(11,12,14-19,21,22)$, and one study that used multiple doses (23). Tandoc et al (15) performed 2 sub studies; one with low doses and one with high doses of added dexamethasone. Analyses of different doses of dexamethasone were selectively performed to assess duration of analgesia and duration of motor block, due to the limited number of studies.

Several approaches were used for brachial plexus block: 8 studies used supraclavicular approach $(10,12,14,17,18,20,21,23), 5$ studies used the interscalene approach $(11,13,15,16,19)$, and one study used an axillary approach (22). Eleven out of 14 studies listed the technique they used to identify the targeted brachial plexus nerves. Five studies listed ultrasound guidance $(10,13,16,18,19)$, nerve stimulator guidance was used in 6 studies $(11,12,14,15,20,22)$, and some studies did not mention the employed techniques $(17,21,23)$ (Table 1).

In this meta-analysis, control groups contained normal saline $(10-20,22,23)$ or tramadol $(21)$ in the injected LA solution without added dexamethasone. LA for brachial nerve block mixed with dexamethasone/other adjuncts included $0.5 \%$ bupivacaine $(14-19,21,23), 0.5 \%$ ropivacaine $(11,16), 0.5 \%$ levobupivacaine $(10,13), 1.5 \%$ mepivacaine (18), 1.5 - $2 \%$ lidocaine $(12,14,22,23)$, and $2 \%$ lidocaine $(17,20)$. Two trials used a combination of 2 LA $(14,17)$. Cummings et al (16) performed 2 comparisons in which LA were either $0.5 \%$ bupivacaine or $0.5 \%$ ropivacaine.

\section{Duration of Analgesia}

Thirteen studies including 982 patients reported duration of analgesia (in minutes). Perineural dexamethasone administration significantly prolonged duration of LA-induced analgesia [SMD $3.90(95 \% \mathrm{Cl}$ : 3.10, $4.70 P<0.00001$ ) $\left.\right|^{2}=94 \%$ ] (Fig. 2c). These effects

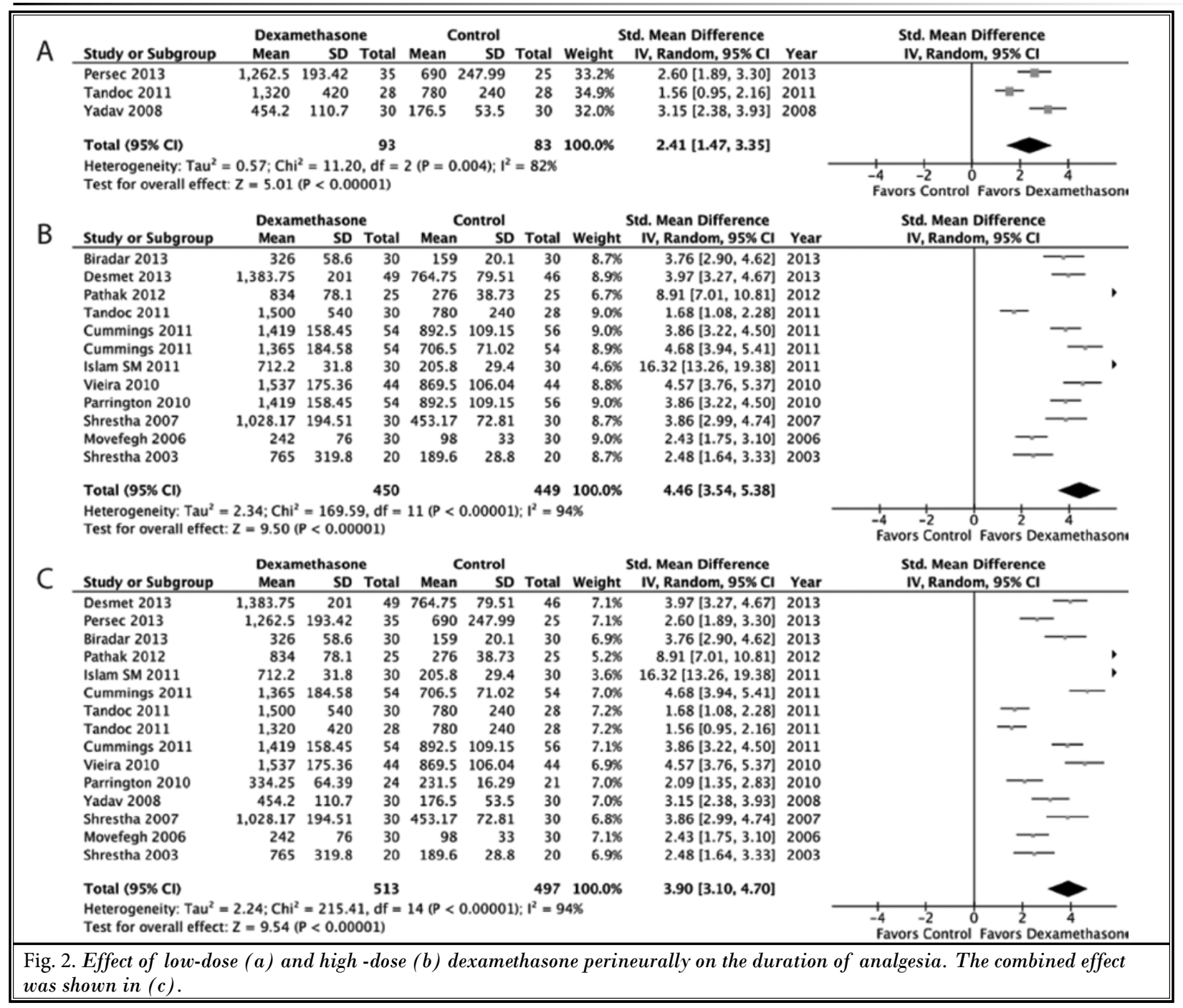


were exhibited in both groups of dexamethasone; 3 studies with low-doses [SMD 2.41 (95\% Cl: 1.47, 3.35 $P=0<0.00001)\left.\right|^{2}=82 \%$ ] (Fig. 2a) and 11 studies with high-doses [SMD $4.46(95 \% \mathrm{Cl} 3.54,5.38 P<0.00001) \mathrm{I}^{2}$ $=94 \%$ ] (Fig. 2b). One study was not included in the specific dose evaluation because there was no separation between low- and high-dose dexamethasone preparations in the study (23).

We examined further if the different LA used interfered with the effect of dexamethasone. The study groups using only one LA at a time were selected $(11,12,15,16,21-23)$. A similar prolonging effect of dexamethasone was also shown among study groups in each LA group. Four studies used $0.5 \%$ bupivacaine $(15,16,21,23)\left[\left.S M D 2.67(95 \% \mathrm{Cl}: 1.65,3.69 P<0.00001)\right|^{2}\right.$ $=91 \%$ ] (Fig. $3 a$ ); 2 studies used $0.5 \%$ ropivacaine $(11,16)$ [SMD $4.31(95 \% \mathrm{Cl}: 3.62,5.00 P<0.00001) \mathrm{I}^{2}=45 \%$ ] (Fig. 3b); and 3 studies used $1.5 \%$ lidocaine $(12,14,22)$ [SMD 4.85 (95\% Cl: 2.24, $7.45 P=0.0003) I^{2}=95 \%$ ] (Fig. 3c). Two studies using $0.5 \%$ levobupivacaine were not analyzed due to limitations in their calculations $(10,13)$.

Different approaches to brachial plexus block were also evaluated. Interscalene $(11,13,15,16,19)$ [SMD 3.37 (95\% Cl: $\left.2.22,4.52 P<0.00001) \mathrm{I}^{2}=94 \%\right]$ and supraclavicular $(10,12,14,17,18,20,21,23)$ [SMD $5.00(95 \% \mathrm{Cl}$ : 3.38, $6.62 P<0.00001) I^{2}=95 \%$ ] approaches provided similar outcomes in terms of duration of analgesia (Figs. $4 a$ and $4 b$, respectively).

\section{Duration of Motor Block}

Duration of motor block was prolonged by added dexamethasone in a pooled analysis of a total of 6 studies in 404 patients $(12,14,15,19,21,22)$ [SMD $2.52(95 \%$ Cl: $1.06,3.98 P=0.0007) l^{2}=97 \%$ ] (Fig. $5 a$ ).

\section{Onset of Sensory and Motor Block}

From 8 studies (435 patients) $(12,14,17,18,20-23)$, perineural dexamethasone injection minimally, but significantly, delayed the onset of sensory block [SMD $-0.49(95 \% \mathrm{Cl}:-0.89,-0.09 P=0.02) \mathrm{I}^{2}=76 \%$ ] (Fig. 5b). Similar delayed onset of motor block [SMD -0.56 (95\% Cl: $-1.13,0.00 P=0.05) I^{2}=87 \%$ ] (Fig. $5 \mathrm{c}$ ) from 7 studies was observed (395 patients) $(12,14,17,18,20,21,22)$.

\section{Pain Scores}

We examined postoperative pain scores during movement at 24 hours [5 study groups, 451 patients $(10,13,16,18,19)$ and 48 hours (3 study groups, 346 patients $(13,16,19)]$ because data from these intervals were readily available. Added dexamethasone treatment exhibited decreased postoperative pain scores at both 24 hours [SMD -1.46 (95\% Cl: $-2.43,-0.50 P=0.003)$

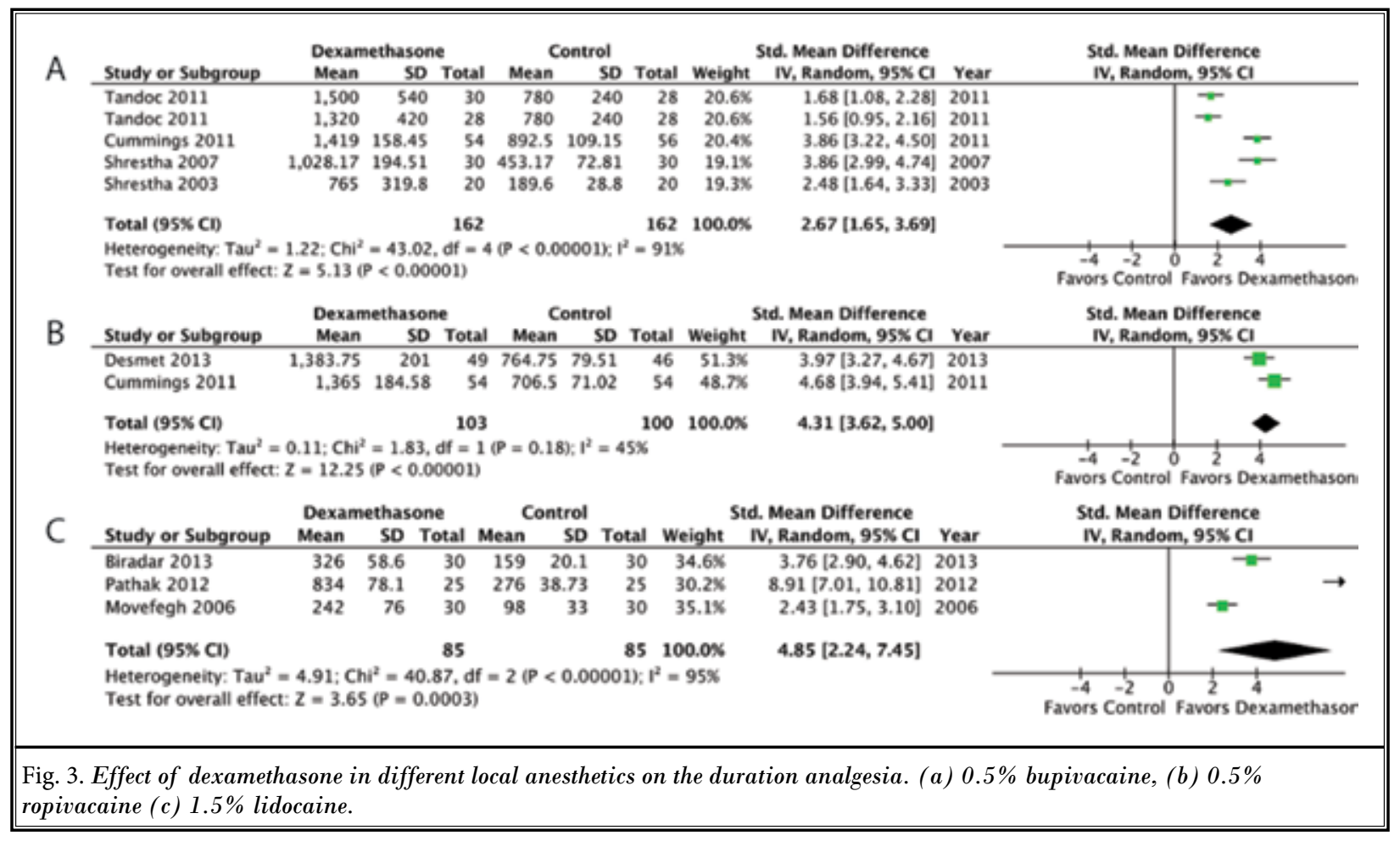


Pain Physician: January/February 2015; 18:1-14

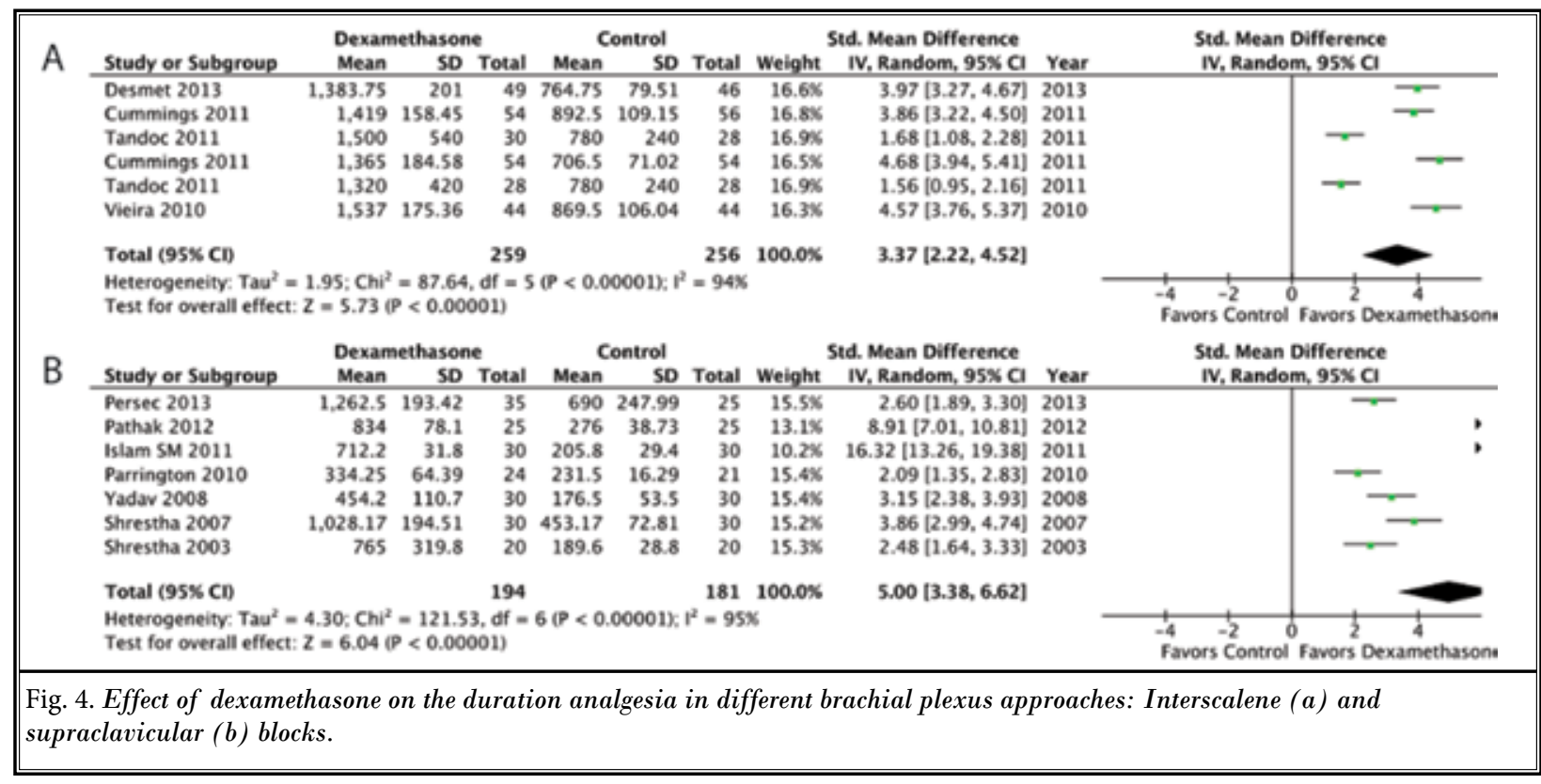

\begin{tabular}{|c|c|c|c|c|c|c|c|c|c|c|c|}
\hline \multirow[t]{10}{*}{$\mathrm{A}$} & Study or Subgroup & \multicolumn{3}{|c|}{ Dexamethasone } & \multicolumn{3}{|c|}{ Control } & \multicolumn{2}{|r|}{ Std. Mean Difference } & \multicolumn{2}{|r|}{$\begin{array}{l}\text { Std. Mean Difference } \\
\text { IV, Random, } 95 \times \mathrm{CI}\end{array}$} \\
\hline & Biradar 2013 & 290.6 & 52.7 & 30 & D 135.5 & 520. & .3 & $30 \quad 14.2$ & $3.83[2.96,4.71]$ & 1) 2013 & 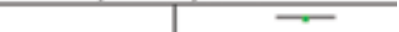 \\
\hline & Pathak 2012 & 376.4 & 39.99 & 25 & $5 \quad 175.2$ & 226.9 & & $25 \quad 13.3$ & $5.81[4.50,7.12]$ & 2012 & $\rightarrow$ \\
\hline & Tandoc 2011 & 2,340 & 2.040 & 28 & 81,500 & 090 & 00 & $28 \quad 14.6$ & $0.53[-0.01,1.06]$ & 2011 & - \\
\hline & Tandoc 2011 & 2.220 & 1.080 & 30 & 01,500 & 090 & $\infty 0$ & $28 \quad 14.6$ & $0.71[0.18,1.24]$ & i) 2011 & 一 \\
\hline & Vieira 2010 & $1,365.5$ & 116.73 & 44 & 4857.5 & 594.6 & & $44 \quad 14.2$ & $4.74[3.91,5.57]$ & 2010 & 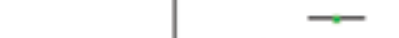 \\
\hline & Shrestha 2007 & 393.03 & 98.96 & 30 & 202.93 & 330.5 & & $30 \quad 14,4$ & $2.56[1.87,3.26]$ & 2007 & $\div$ \\
\hline & Movefegh 2006 & 310 & 817 & 30 & 393.03 & 398.9 & & $30 \quad 14.6$ & $-0.14[-0.65,0.37]$ & 2006 & \\
\hline & Total $(95 \% \mathrm{CD})$ & & & 217 & & & & 15100.0 & $2.52[1.06,3.98]$ & & \\
\hline & $\begin{array}{l}\text { Heterogeneity: Tau }= \\
\text { Test for overall effect: }\end{array}$ & $\begin{array}{l}3.73 ; C_{i} \\
z=3.38\end{array}$ & $\begin{array}{l}P^{2}=198 \\
(P=0.0\end{array}$ & .26, df & $f=6(P<$ & $<0.000$ & Do1): $r^{2}=$ & $=97 x$ & & & $\begin{array}{ccccc}-1 & 1 & 1 & 1 & 1 \\
-4 & -2 & 0 & 2 & 4 \\
\text { Favors Control Favors Dexamethasona }\end{array}$ \\
\hline \multirow{12}{*}{ B } & & \multicolumn{3}{|c|}{ Dexamethasone } & \multicolumn{3}{|c|}{ Control } & \multirow{2}{*}{\multicolumn{2}{|c|}{$\begin{array}{c}\text { Std. Mean Difference } \\
\text { Weight } \quad \text { IV, Random, 95* C }\end{array}$}} & & \multirow{2}{*}{$\begin{array}{l}\text { Std. Mean Difference } \\
\text { IV, Random, 95\% CI }\end{array}$} \\
\hline & Study or Subgroup & Mean & SD & Total & Mean & SD & Total & & & Year & \\
\hline & Biradar 2013 & 13.4 & 2.8 & 30 & 16 & 2.3 & 30 & $12.7 \%$ & $-1.00[-1.54,-0.46]$ & 2013 & $\mp$ \\
\hline & Pathak 2012 & 5.92 & 2.8 & 25 & 6.62 & 2.95 & 25 & $12.5 \%$ & $-0.23[-0.79,0.32]$ & 2012 & 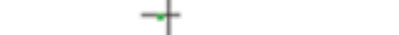 \\
\hline & Islam SM 2011 & 9.89 & 1.97 & 30 & 11.642 & 2.19 & 30 & $12.8 \times$ & $-0.83[-1.36,-0.30]$ & 2011 & $\sim$ \\
\hline & Parrington 2010 & 9 & 5 & 24 & 10 & 4 & 21 & 12.18 & $-0.22[-0.80,0.37]$ & 2010 & 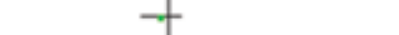 \\
\hline & Yadav 2008 & 3.8 & 1.8 & 30 & 4.6 & 1.1 & 30 & $12.9 \%$ & $-0.53[-1.04,-0.01]$ & 2008 & $\rightarrow$ \\
\hline & Shrestha 2007 & 16.76 & 2.34 & 30 & 18.472 & 2.03 & 30 & $12.8 \%$ & $-0.77[-1.30,-0.24]$ & 2007 & \\
\hline & Movefegh 2006 & 14 & 5 & 30 & 11 & 4 & 30 & $12.9 \%$ & $0.65[0.13,1.17]$ & 2006 & - \\
\hline & Shrestha 2003 & 14.5 & 2.1 & 20 & 18.15 & 4.25 & 20 & 11.36 & $-1.07[-1.73,-0.40]$ & 2003 & \\
\hline & Total $(95 \% \mathrm{Cl})$ & & & 219 & & & 216 & $100.0 \%$ & $-0.49[-0.89,-0.09]$ & & \\
\hline & $\begin{array}{l}\text { Heterogeneity: Tau }{ }^{2} \\
\text { Test for overall effect }\end{array}$ & $\begin{array}{l}=0.25 ; C h \\
z=2.42\end{array}$ & $\begin{array}{l}h^{2}=29 \\
2(P=0\end{array}$ & $\begin{array}{l}9.24, \mathrm{df} \\
.02)\end{array}$ & If $=7(\mathrm{P}$ & $=0.00$ & 001): $\mathrm{I}^{2}$ & $2=76 x$ & & & $\begin{array}{ccccc}-1 & 1 & 1 & 1 & 1 \\
-4 & -2 & 0 & 2 & 4 \\
\text { Dexamethasone } & \text { Favors Control }\end{array}$ \\
\hline \multirow{11}{*}{ C } & & \multicolumn{3}{|c|}{ Dexamethasone } & \multicolumn{3}{|c|}{ Control } & \multicolumn{2}{|c|}{ Std. Mean Difference } & & \multirow{2}{*}{$\begin{array}{l}\text { Std. Mean Difference } \\
\text { IV, Random, } 95 \% \mathrm{Cl}\end{array}$} \\
\hline & Study or Subgroup & Mean & SD & Total & Mean & SD 1 & Total & Weight & IV, Random, 95\% CI & Year & \\
\hline & Biradar 2013 & 16 & 2.7 & 30 & 18.7 & 2.8 & 30 & $14.4 x$ & $-0.97[-1.51,-0.43]$ & 2013 & $\mp$ \\
\hline & Pathak 2012 & 15.8 & 5.6 & 25 & 16.6 & 5.11 & 25 & $14.3 \%$ & $=0.15[-0.70,0.41]$ & 2012 & \\
\hline & Islam SM 2011 & 11.09 & 1.28 & 30 & 13.32 & 0.98 & 30 & $13.8 \%$ & $-1.93[-2.55,-1.31]$ & 2011 & $\longrightarrow$ \\
\hline & Parrington 2010 & 8 & 3 & 24 & 8 & 3 & 21 & $14.0 \%$ & $0.00[-0.59,0.59]$ & 2010 & \\
\hline & Yadav 2008 & 6 & 2.1 & 30 & 7.7 & 2 & 30 & $14.5 \%$ & $-0.82[-1.35,-0.29]$ & 2008 & 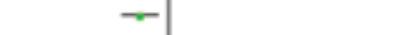 \\
\hline & Shrestha 2007 & 12.9 & 1.49 & 30 & 13.931 & 1.66 & 30 & $14.5 \%$ & $-0.64[-1.16,-0.12]$ & 2007 & - \\
\hline & Movefegh 2006 & 26 & 7 & 30 & 22 & 8 & 30 & $14.5 \%$ & $0.53[0.01,1.04]$ & 2006 & \\
\hline & Total $(95 \% \mathrm{Cl})$ & & & 199 & & & 196 & $100.0 \%$ & $-0.56[-1.13,0.00]$ & & \\
\hline & $\begin{array}{l}\text { Heterogeneity, Tau } \\
\text { Test for overall effect }\end{array}$ & $\begin{array}{l}=0.50 ; C h \\
z=1.95\end{array}$ & $\begin{array}{l}h^{2}=44 \\
5(P=0\end{array}$ & $\begin{array}{l}\text { 4.62, df } \\
. .05)\end{array}$ & $f=6(P$ & $<0.00$ & 0001): 1" & $1^{2}=87 \%$ & & Fave & 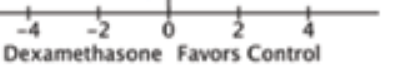 \\
\hline
\end{tabular}


$\mathrm{I}^{2}=95 \%$ ] (Fig. 6a) and at 48 hours [SMD $-1.20(95 \% \mathrm{Cl}$ : $-2.26,-0.13 P=0.03) I^{2}=95 \%$ ] (Fig. 6b).

\section{Opioid Consumption}

Postoperatively administered opioids used in the included studies were as follows: hydrocodone (15), oxycodone $(16,19)$, and oral morphine (18). Opioid consumption was assessed at 24 hours and 48 hours postoperatively. Similar opioid consumptions were reported from both dexamethasone-injected groups and control groups from 2 studies (total 133 patients) $(18,19)$ at the 24 hour period [SMD $-1.74(95 \% \mathrm{Cl}:-5.27,1.79 P=$ $0.33) I^{2}=98 \%$ ] (Fig. 7a). At 48 hours following surgery, perineural added dexamethasone was associated with a substantially lower opioid consumption from 174 patients in 2 study groups $(15,19)$ [SMD $-2.97(95 \% \mathrm{Cl}$ : $-4.17,-1.76 P<0.00001)\left.\right|^{2}=88 \%$ ] (Fig. 7b).

\section{Complications}

Certain of the included studies further investigated complications following brachial plexus blocks. There were 4 studies which provided results eligible for analysis $(11,13,17,18)$. Addition of perineural adjunctive dexamethasone did not alter the incidence of complications following the regional blocks and/or general anesthesia, including the incidence of nausea $(13,18)$ [SMD -0.01 (95\% Cl: $-0.16,0.15 P=0.93) \mathrm{l}^{2}=0 \%$ ], hoarseness $(11,13,17)$ [SMD $-0.01(95 \% \mathrm{Cl}:-0.14,0.12 P$

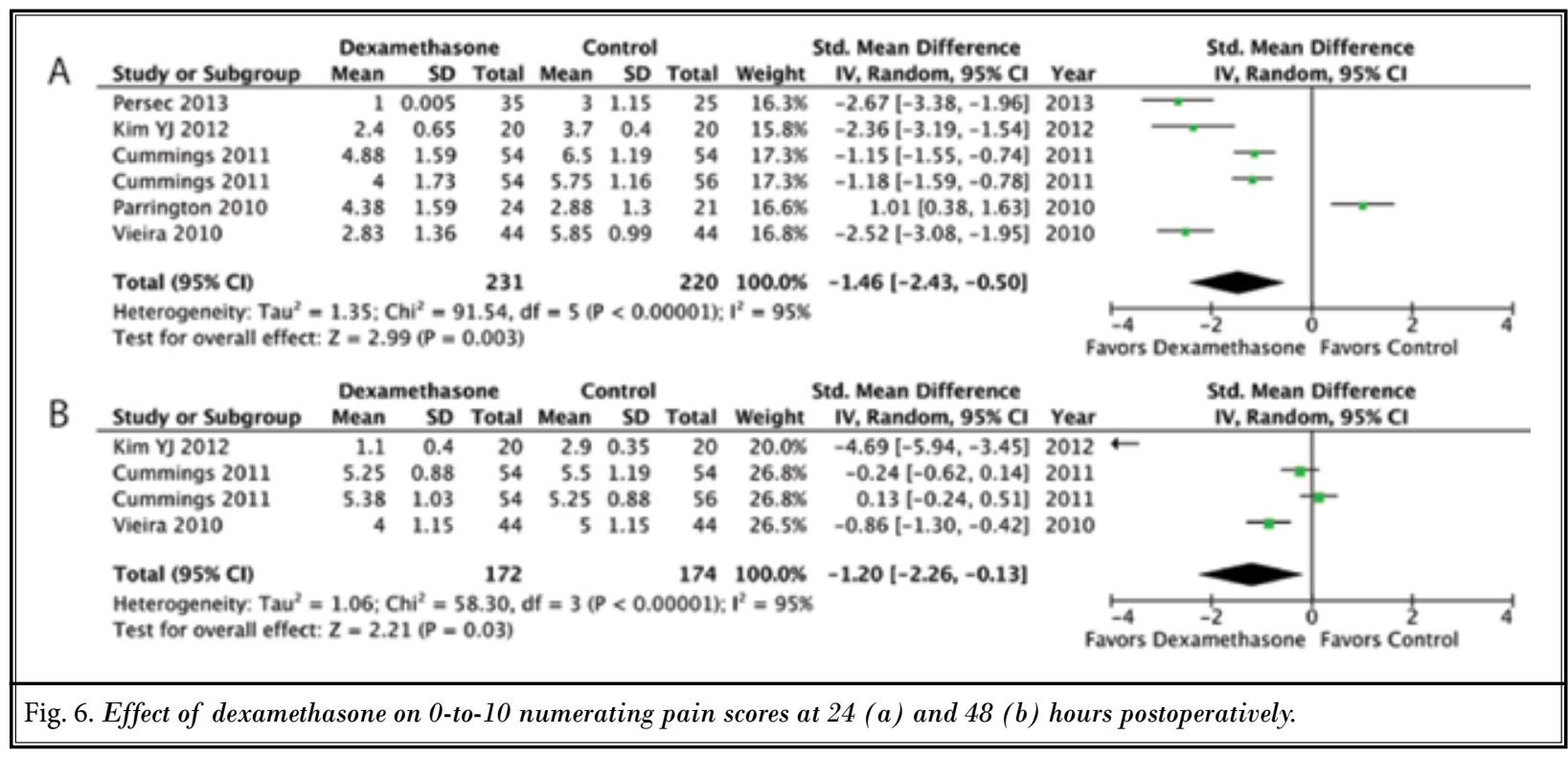

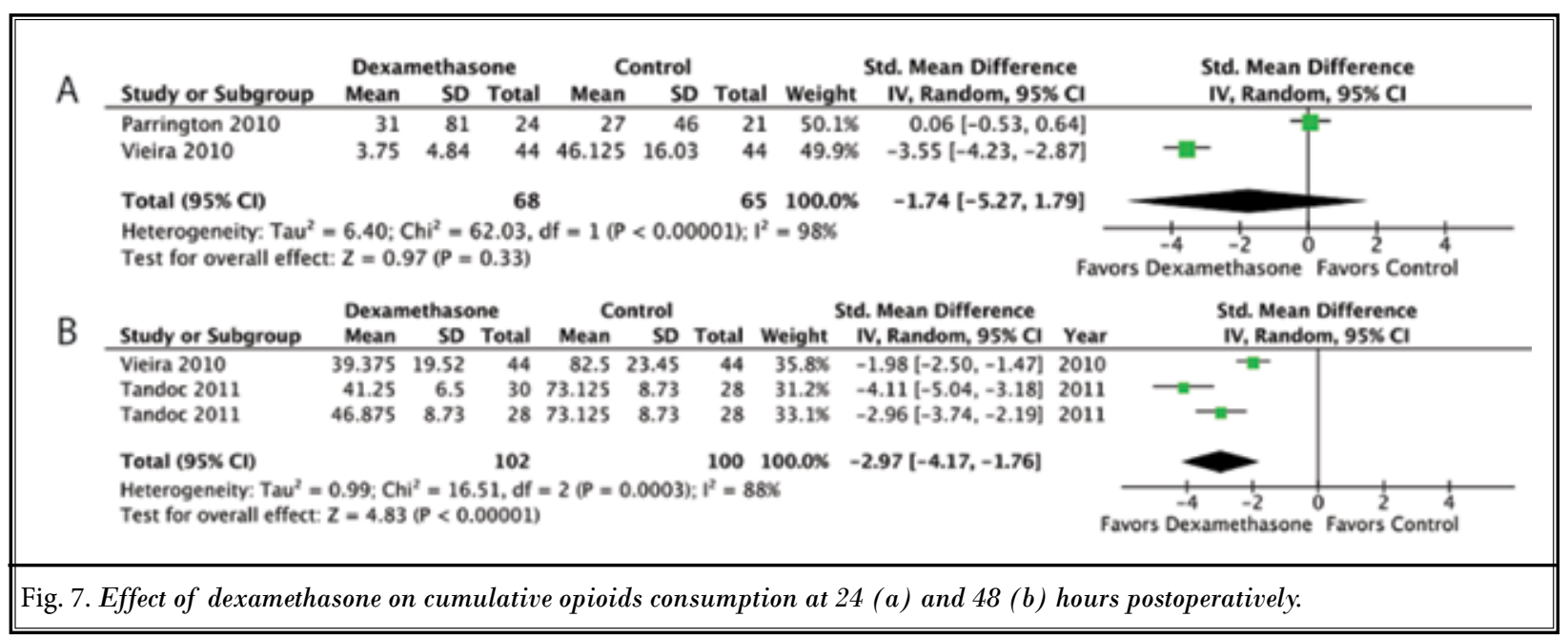


Pain Physician: January/February 2015; 18:1-14

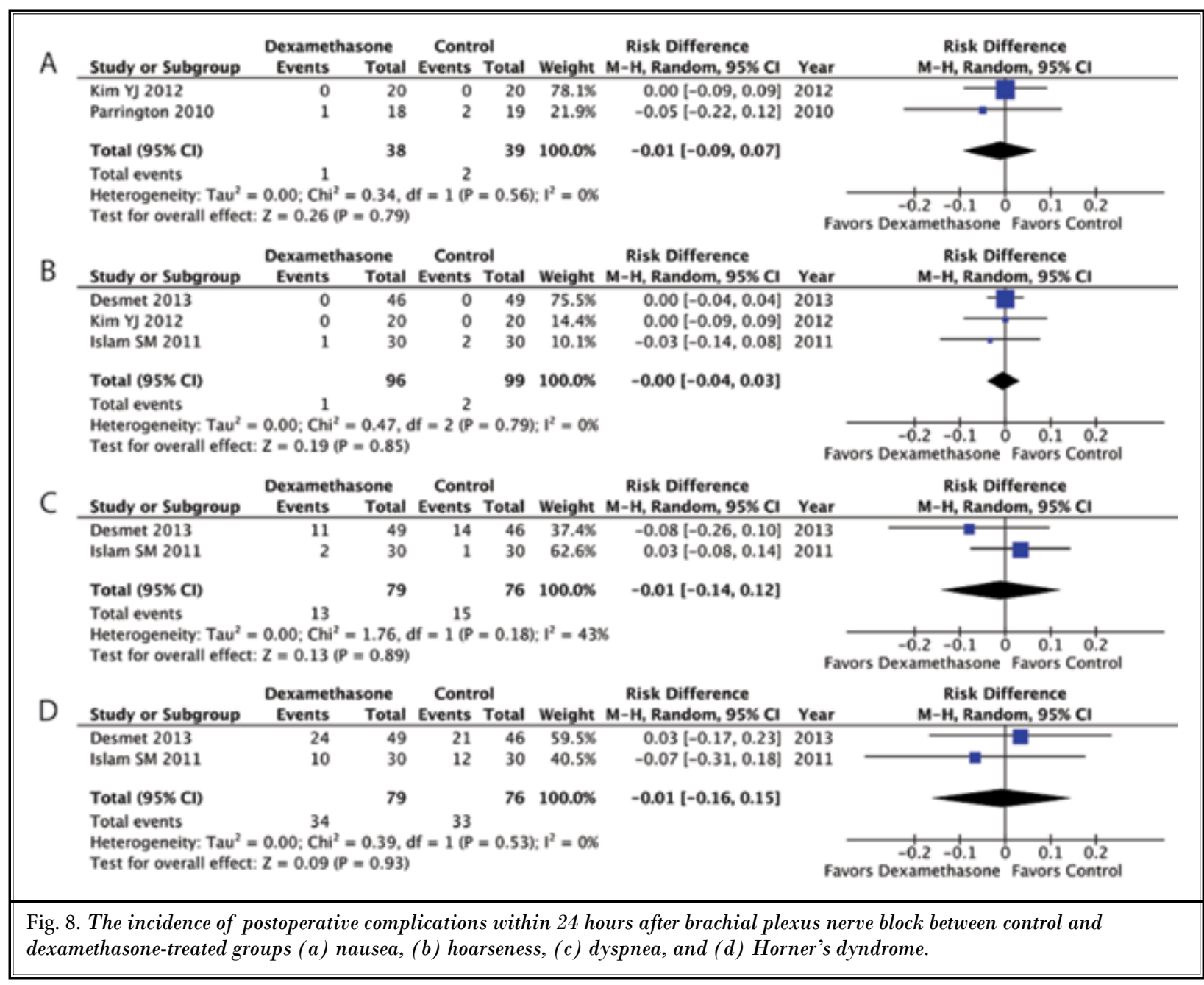

$=0.89) \mathrm{I}^{2}=43 \%$, dyspnea $(11,17)$ [SMD $0.00(95 \% \mathrm{Cl}$ : $-0.04,0.03 P=0.85) I^{2}=0 \%$, and Horner's syndrome $(11,17)$ [SMD $-0.01(95 \% \mathrm{Cl}:-0.09,0.07 P=0.79) \mathrm{I}^{2}=0 \%$ ] (Figs. 8a, 8b, 8c, and 8d, respectively).

\section{Discussion}

Our findings have identified a significant beneficial postoperative effect of adding perineural dexamethasone to LA for brachial plexus blocks in several regards: duration of analgesia, pain scores, and opioid consumption. Both low (4 - $5 \mathrm{mg}$ ) and high doses (8 $-10 \mathrm{mg}$ ) of dexamethasone used in perineural adjunctive application comparably prolonged the duration of brachial plexus analgesia, regardless of different LA types and brachial block approaches. Although only 3 studies were analyzed and heterogeneity was high, low doses of dexamethasone were shown to adequately diminish postoperative pain. A recent meta-analysis, which focused on the anesthetics based on different durations of action combined with dexamethasone in brachial nerve blocks, also showed a favorable effect of dexamethasone on sensory block postoperatively (50).

By adding dexamethasone to LA, pain scores were lowered at 24 hours and 48 hours postoperatively corresponding to less opioid consumption over those 24 - 48 hours. This opioid-sparing effect could potentially translate into a reduction in opioid-related adverse events, including nausea, vomiting, pruritis, and urinary retention, constipation, and respiratory depression. Nevertheless, with the somewhat limited numbers from only 2 studies, opioid consumption in the first 24 hours appeared not to be affected by dexamethasone addition. Choi et al (50) reports no difference in opioid consumption up to 72 hours. However, we analyzed 24- and 
48-hour time intervals separately, which provided more detailed results. In other studies, the overall effects of intravenous (and not perineural) dexamethasone were found to decrease pain scores and opioid consumption postoperatively at 24 hours from a large number of pooled studies $(5,6)$. More studies could elaborate whether or not there is an opioid-sparing effect of perineural dexamethasone. Dexamethasone is believed to mediate its analgesic effects by increasing the activity of inhibitory $\mathrm{K}$ channels on nociceptive C-fibers $(51,52)$. Another theory is that glucocorticoids produce modest amounts of vasoconstriction, which might reduce LA absorption, hence prolonging LA-nerve contact time $(22,53)$. However, other authors believe its analgesic effect is moderated exclusively or largely systemically $(5,6)$.

Interestingly, perineurally administered dexamethasone added to LA delayed the latency and prolonged the recovery of motor block after brachial plexus block, similar to the analysis from Choi et al (50). We additionally found a delayed latency of onset of sensory and motor blocks from dexamethasone administration. These undesirable effects of added perineural dexamethasone may limit its clinical usefulness. However, these results were shown mainly when high-dose dexamethasone was employed. A dose-response study of these parameters may facilitate greater comprehension and provide a rationale for selecting an appropriate dose of perineural dexamethasone.

The technique of brachial plexus block may result in neurological complications including respiratory distress, Horner's syndrome, and recurrent laryngeal nerve injury $(54,55)$. Several included studies confirmed the safety of perineural dexamethasone administration in terms of not inducing the development of nausea, vomiting, numbness/tingling, respiratory distress, hoarseness, and Horner's syndrome $(11,13,17,18)$. We found that adding perineural dexamethasone for brachial plexus blocks likewise did not mitigate against the development of these consequences as the incidence of dyspnea, hoarseness, and Horner's syndrome were shown to be similar in both control and dexamethasonetreated groups. Although not analyzed in our study due to inadequate patient numbers, these complications are reported as comparable in both control and dexamethasone-injected groups; recurrent laryngeal nerve blocks $(13,17)$, neurological disabilities (13), phrenic nerve palsy (14), pruritus (14), and drowsiness (14). In 2 of our included studies, patients were followed-up for 2 weeks without any persistent symptoms of paresthesias and with no cases of paralysis in the operated-upon upper limbs $(16,18)$. However, the only significant difference was that the dexamethasone-injected group had excessively prolonged nerve blocks postoperatively as a side effect, compared with the control group. Furthermore, the known salubrious effect of dexamethasone in PONV was not clearly demonstrated in our analysis, while IV dexamethasone significantly improved PONV in a previous analysis (7).

As for concern for neurotoxicity from perineurallyadded dexamethasone, there is a clinical controversy regarding limiting the dose added to LA to $1-2 \mathrm{mg}$ (2). Results from animal studies demonstrated no long-term alteration in function or histology of nerve fibers as a result of applying $0.8-3.2 \mathrm{mg}$ dexamethasone over exposed peripheral nerves (56). The topical application of $0.4 \%$ dexamethasone on a rat sciatic nerve significantly reduced nerve blood flow but did not cause ischemic changes (53). Another in-vitro study revealed that incubation of isolated sensory neurons with only dexamethasone equivalent to the high-dose concentrations used in studies that we reviewed caused less neuronal cell death than ropivacaine alone. However, 24 hour exposure to higher doses of dexamethasone added to ropivacaine enhanced the ropivacaine-induced neurotoxicity (28). In a recently published editorial, Williams et al (57) suggested eliminating studies exploring perineural dexamethasone at doses $8 \mathrm{mg}$ and greater. High doses of dexamethasone are not proven to be more effective in many studies, but rather appear to be cytotoxic when applied in cultured neurons which is also consistent with our conclusion (57). We included all published studies that used $4 \mathrm{mg}$ dexamethasone and confirmed that lowering the dose of dexamethasone should be a trend in any future use of perineural anesthesia. Even applied locally, dexamethasone, by being lipid soluble, could produce corticosteroid effects systemically which may be avoided by using lower doses. We could conclude through our systematic meta-analysis of brachial plexus perineural blocks that a $4 \mathrm{mg}$ dose does not produce statistically significant differences compared with higher doses in term of neural block duration, pain, and motor block. Results of our meta-analysis suggest that a single perineural dexamethasone application is safe in the short-term postoperative assessment. However, more dose-response studies are encouraged in order to clarify the sufficient and safe, yet effective, concentration of perineural dexamethasone used as an adjuvant to LA administration.

Previous systematic reviews on the effects of IV 
dexamethasone have demonstrated significant improvements in postoperative analgesia $(5,6)$. Intravenous dexamethasone was shown to delay time to first analgesic rescue dosing $(5,6)$. However, De Oliveira et al (5) found that only low-dose IV dexamethasone specifically prolonged time to first analgesic requirement from a total of 4 studies. IV dexamethasone also decreased pain scores at $0-4$ hours, 24 hours, and 48 hours postoperatively $(5,6)$. Opioid consumption was reduced by IV dexamethasone at 2 hours and 24 hours postoperatively in the systematic review of Waldron et al (6), but the study time period was not specified by De Oliveira et al (5). A meta-analysis from Choi at al (50) followed dexamethasone's effects on brachial plexus blocks and pointed out a benefit in analgesia duration. We included 4 more studies in our analysis which demonstrated similar benefits. Furthermore, we captured latency of sensory and motor block and postoperative pain scores after brachial plexus dexamethasone administration. Results of both meta-analyses showed that perineural adjunctive injection of dexamethasone significantly improved the postoperative duration of analgesia and prolonged the duration of motor block. Although the number of pooled studies was different between our review of brachial plexus perineural dexamethasone and the previous studies on IV dexamethasone, postoperative analgesia was enhanced significantly by dexamethasone added by either route. These findings beg the question as to the actual mechanism of action of dexamethasone as a perioperative analgesic. Whether or not the agent produces analgesia systemically or regionally remains unknown at present.

A reasonable assessment of which method (systemic or regional) is more efficient might be drawn from a direct comparison of these respective routes in the same study. There are increasing numbers of studies which performed a comparison between IV and perineural routes of dexamethasone using the same control group; 2 in a brachial block model $(11,58)$, and one in an epidural model (59). However, the advantages of perineural over IV dexamethasone from these reports are controversial. Kawanishi et al (58) reported improvement of postoperative analgesia using 4-mg dexamethasone perineurally compared to IV dexamethasone injection used in an interscalene block model. However, Desmet et al (11) reported comparable effects of dexamethasone in terms of duration of analgesia and pain scores for up to $24-48$ hours for both administration routes. Due to the limited number of studies assessing this issue, more trials are required for systematic analysis.

Our meta-analysis was limited by the number of studies assessing certain parameters having high heterogeneity. Different definitions used for the measurements of certain parameters such as the duration of analgesia and duration of motor block were also limitations. Most of the included studies calculated the duration of analgesia/motor block as being from the onset time of dexamethasone injection or from the time of attaining complete analgesia intraoperatively, except Tandoc et al (15) who assessed these factors after postanesthesia care unit (PACU) discharge. However, data from each study demonstrated similar favorable effects of adding perineural dexamethasone to LA used for brachial plexus blocks. Although heterogeneity was high, removing the Tandoc et al (15) study did not affect it.

Another limitation was the varying types of LA used in these studies included for our review. Here we have shown that the benefits of dexamethasone on duration of analgesia was similarly identified using different LA types; $0.5 \%$ bupivacaine, $0.5 \%$ ropivacaine, and $1.5 \%$ lidocaine. Two studies using $0.5 \%$ levobupivacaine also identified a positive analgesic effect of adding dexamethasone in terms of reducing pain scores $(10,13)$. However, the various additional adjuncts (neostigmine, epinephrine, clonidine) added to the LA or even using a mixture of 2 LA may impact or limit our overall interpretation of the results. As for the different approaches used for brachial block, interscalene and supraclavicular approaches were previously shown to be similarly effective and safe for shoulder surgery (60). Similarly, we found positive results on the duration of analgesia of adding dexamethasone using both techniques. The type of surgery, dose of dexamethasone, analgesics used other than opioids, and completeness of reported outcomes also limited our analysis.

\section{Conclusion}

In conclusion, we found a substantial clinical and statistical effect of adding perineural dexamethasone to LA used for brachial plexus blocks in terms of a prolongation of duration of analgesia as well as on the reduction of pain scores and opioid consumption postoperatively. Our results showed that low-dose dexamethasone is sufficient to enhance analgesia associated with brachial plexus blocks when applied perineurally. Nevertheless, this analgesic effect of dexamethasone was associated with delayed onset of anesthetic sensory blockade and delayed recov- 
ery from motor block. More studies are required to evaluate the practical benefits and clinical safety of perineural dexamethasone as a LA adjunct. Furthermore, direct comparisons between perineural and IV dexamethasone administration would also provide information regarding the optimal route of administration and a possible clarification of the mechanism of action of this adjuvant.

\section{References}

1. Axelsson K, Gupta A. Local anaesthetic adjuvants: Neuraxial versus peripheral nerve block. Curr Opin Anaesthesiol 2009; 22:649-654.

2. Brummett $\mathrm{CM}$, Williams BA. Additives to local anesthetics for peripheral nerve blockade. Int Anesthesiol Clin 2011; 49:104-116.

3. Khafagy HF, Refaat Al, El-Sabae $\mathrm{HH}$ Youssif MA. Efficacy of epidural dexamethasone versus fentanyl on postoperative analgesia. J Anesth 2010; 24:531-536.

4. Pinto RZ, Maher CG, Ferreira ML, Hancock M, Oliveira VC, McLachlan AJ, Koes $\mathrm{B}$, Ferreira PH. Epidural corticosteroid injections in the management of sciatica: A systematic review and meta-analysis. Ann Intern Med 2012; 157:865-877.

5. De Oliveira GS Jr, Almeida MD, Benzon HT, McCarthy RJ. Perioperative single dose systemic dexamethasone for postoperative pain: A meta-analysis of randomized controlled trials. Anesthesiology 2011; 115:575-588.

6. Waldron $\mathrm{NH}$, Jones $\mathrm{CA}$, Gan TJ, Allen TK, Habib AS. Impact of perioperative dexamethasone on postoperative analgesia and side-effects: Systematic review and meta-analysis. $\mathrm{Br}$ J Anaesth 2013; 110:191-200.

7. De Oliveira GS Jr, Castro-Alves LJ, Ahmad S, Kendall MC, McCarthy RJ. Dexamethasone to prevent postoperative nausea and vomiting: An updated meta-analysis of randomized controlled trials. Anesth Analg 2013; 116:58-74.

8. Lauretti GR, Rizzo CC, Mattos AL, Rodrigues SW. Epidural methadone results in dose-dependent analgesia in cancer pain, further enhanced by epidural dexamethasone. Br] Cancer 2013; 108:259-264.

9. Naghipour B, Aghamohamadi D, Azarfarin R, Mirinazhad M, Bilehjani E, Abbasali D, Golzari SE. Dexamethasone added to bupivacaine prolongs duration of epidural analgesia. Middle East ] Anesthesiol 2013; 22:53-57.

10. Persec J, Persec Z, Kopljar M, Zupcic M, Sakic L, Zrinjscak IK, Marinic DK. Lowdose dexamethasone with levobupivacaine improves analgesia after supraclavicular brachial plexus blockade. Int Orthop 2014; 38:101-105.
11. Desmet M, Braems H, Reynvoet M, Plasschaert S, Van Cauwelaert J, Pottel H, Carlier S, Missant C, Van de Velde M. I.V. and perineural dexamethasone are equivalent in increasing the analgesic duration of a single-shot interscalene block with ropivacaine for shoulder surgery: A prospective, randomized, placebo-controlled study. Br J Anaesth 2013; 111:445-452.

12. Biradar PA, Kaimar P, Gopalakrishna K Effect of dexamethasone added to lidocaine in supraclavicular brachial plexus block: A prospective, randomised, double-blind study. Indian J Anaesth 2013; 57:180-184.

13. Kim YJ, Lee GY, Kim DY, Kim CH, Baik HJ, Heo S. Dexamathasone added to levobupivacaine improves postoperative analgesia in ultrasound guided interscalene brachial plexus blockade for arthroscopic shoulder surgery. Korean J Anesthesiol 2012; 62:130-134

14. Pathak RG, Satkar AP, Khade RN. Supraclavicular brachial plexus block with and without dexamethasone - a comparative study. International Journal of Scientific and Research Publications 2012; 2:1-7.

15. Tandoc MN, Fan L, Kolesnikov S, Kruglov A, Nader ND. Adjuvant dexamethasone with bupivacaine prolongs the duration of interscalene block: A prospective randomized trial. J Anesth 2011; 25:704-709.

16. Cummings KC 3 rd, Napierkowski DE, Parra-Sanchez I, Kurz A, Dalton JE, Brems J, Sessler DI. Effect of dexamethasone on the duration of interscalene nerve blocks with ropivacaine or bupivacaine. $\mathrm{Br}$ J Anaesth 2011; 107:446-453.

17. Islam SM, Hossain MHMD, Maruf AA. Effect of addition of dexamethasone to local anaesthetics in supraclavicular brachial plexus block. Journal of Armed Forces Medical College 2011;7:11-14.

18. Parrington SJ, O'Donnell D, Chan V, Brown-Shreves D, Subramanyam R, Qu $M$, Brull R. Dexamethasone added to mepivacaine prolongs the duration of analgesia after supraclavicular brachial plexus blockade. Regional Anesthesia and Pain Medicine 2010; 35:422-426.

19. Vieira PA, Pulai I, Tsao GC, Manikantan P, Keller B, Connelly NR. Dexamethasone with bupivacaine increases duration of analgesia in ultrasound-guided interscalene brachial plexus blockade. Eur J Anaesthesiol 2010; 27:285-288.

20. Yadav RK, Sah BP, Kumar P, Singh SN. Effectiveness of addition of neostigmine or dexamethasone to local anaesthetic in providing perioperative analgesia for brachial plexus block: A prospective, randomized, double blinded, controlled study. Kathmandu Univ Med J 2008; 6:302-309.

21. Shrestha BR, Maharjan SK, Shrestha $S$, Gautam B, Thapa C, Thapa PB, Joshi MR. Comparative study between tramadol and dexamethasone as an admixture to bupivacaine in supraclavicular brachial plexus block. JNMA J Nepal Med Assoc 2007; 46:158-164.

22. Movafegh A, Razazian M, Hajimaohamadi F, Meysamie A. Dexamethasone added to lidocaine prolongs axillary brachial plexus blockade. Anesth Analg 2006; 102:263-267.

23. Shrestha BR, Maharjan SK, Tabedar S. Supraclavicular brachial plexus block with and without dexamethasone - A comparative study. Kathmandu University Medical Journal 2003; 1:158-160.

24. Fredrickson Fanzca MJ, Danesh-Clough TK, White R. Adjuvant dexamethasone for bupivacaine sciatic and ankle blocks: Results from 2 randomized placebo-controlled trials. Reg Anesth Pain Med 2013; 38:300-307.

25. Aggarwal V, Singla M, Rizvi A, Miglani S. Comparative evaluation of local infiltration of articaine, articaine plus ketorolac, and dexamethasone on anesthetic efficacy of inferior alveolar nerve block with lidocaine in patients with irreversible pulpitis. J Endod 2011; 37:445-449.

26. Jürgens TP, Müller P, Seedorf H, Regelsberger J, May A. Occipital nerve block is effective in craniofacial neuralgias but not in idiopathic persistent facial pain. J Headache Pain 2012; 13:199-213.

27. Tan PH, Liu K, Peng $\mathrm{CH}$, Yang LC, Lin CR, Lu CY. The effect of dexamethasone on postoperative pain and emesis after intrathecal neostigmine. Anesth Analg 2001; 92:228-232.

28. Williams BA, Hough KA, Tsui BY, Ibinson JW, Gold MS, Gebhart GF. Neurotoxic- 
ity of adjuvants used in perineural anesthesia and analgesia in comparison with ropivacaine. Reg Anesth Pain Med 2011; 36:225-230.

29. Liberati A, Altman DG, Tetzlaff J, Mulrow C, Gøtzsche PC, loannidis JP, Clarke M, Devereaux PJ, Kleijnen J, Moher D. The PRISMA statement for reporting systematic reviews and meta-analyses of studies that evaluate healthcare interventions: Explanation and elaboration. BM] 2009; 339:b270o.

30. Korff MV, Saunders K, Thomas Ray G, Boudreau D, Campbell C, Merrill J, Sullivan MD, Rutter CM, Silverberg MJ, Banta-Green C, Weisner C. De facto longterm opioid therapy for noncancer pain. Clin J Pain 2008; 24:521-527.

31. Hozo SP, Djulbegovic B, Hozo I. Estimating the mean and variance from the median, range, and the size of a sample. BMC Medical Research Methodology 2005; 5:13.

32. Oremus $M$, Wolfson $C$, Perrault A, Demers L, Momoli F, Moride Y. Interrater reliability of the modified Jadad quality scale for systematic reviews of Alzheimer's disease drug trials. Dement Geriatr Cogn Disord 2001; 12:232-236.

33. Jiang N, Lin QR, Diao XC, Wu L, Yu B. Surgical versus nonsurgical treatment of displaced intra-articular calcaneal fracture: A meta-analysis of current evidence base. Int Orthop 2012; 36:1615-1622.

34. DerSimonian R, Laird N. Meta-analysis in clinical trials. Control Clin Trials 1986; 7:177-188.

35. Bani-Hashem N, Hassan-Nasab B, Pour EA, Maleh PA, Nabavi A, Jabbari A. Addition of intrathecal dexamethasone to bupivacaine for spinal anesthesia in orthopedic surgery. Saudi J Anaesth 2011; 5:382-386.

36. Cardoso MM, Leite AO, Santos EA, Gozzani JL, Mathias LA. Effect of dexamethasone on prevention of postoperative nausea, vomiting and pain after caesarean section: A randomised, placebo-controlled, double-blind trial. Eur ] Anaesthesiol 2013; 30:102-105.

37. Bergeron SG, Kardash KJ, Huk OL, Zukor DJ, Antoniou J. Perioperative dexamethasone does not affect functional outcome in total hip arthroplasty. Clin Orthop Relat Res 2009; 467:1463-1467.

38. Ikeuchi M, Kamimoto Y, Izumi M, Fukunaga K, Aso K, Sugimura N, Yokoyama $M$, Tani T. Effects of dexamethasone on local infiltration analgesia in total knee arthroplasty: A randomized controlled trial. Knee Surg Sports Traumatol Arthrosc
2014; 22:1638-1643.

39. Movafegh A, Soroush AR, Navi A, Sadeghi $\mathrm{M}$, Esfehani F, Akbarian-Tefaghi $\mathrm{N}$. The effect of intravenous administration of dexamethasone on postoperative pain, nausea, and vomiting after intrathecal injection of meperidine. Anesth Analg 2007; 104:987-989.

40. Wang YL, Tan PP, Yang CH, Tsai SC, Chung HS. Epidural dexamethasone reduces the incidence of backache after lumbar epidural anesthesia. Anesth Analg 1997; 84:376-378.

41. Bigat Z, Boztug N, Hadimioglu N, Cete $N$, Coskunfirat N, Ertok E. Does dexamethasone improve the quality of intravenous regional anesthesia and analgesia? A randomized, controlled clinical study. Anesth Analg 2006; 102:605-609.

42. Jankovic RJ, Visnjic MM, Milic DJ, Stojanovic MP, Djordjevic DR, Pavlovic MS. Does the addition of ketorolac and dexamethasone to lidocaine intravenous regional anesthesia improve postoperative analgesia and tourniquet tolerance for ambulatory hand surgery? Minerva Anestesiol 2008; 74:521-527.

43. Tolver MA, Strandfelt P, Bryld EB, Rosenberg J, Bisgaard T. Randomized clinical trial of dexamethasone versus placebo in laparoscopic inguinal hernia repair. $\mathrm{Br}$ J Surg 2012; 99:1374-1380.

44. Holte K, Werner MU, Lacouture PG, Kehlet $\mathrm{H}$. Dexamethasone prolongs local analgesia after subcutaneous infiltration of bupivacaine microcapsules in human volunteers. Anesthesiology 2002; 96:1331-1335.

45. Kopacz DJ, Lacouture PG, Wu D, Nandy $P$, Swanton R, Landau C. The dose response and effects of dexamethasone on bupivacaine microcapsules for intercostal blockade ( $T_{9}$ to $\left.T_{11}\right)$ in healthy volunteers. Anesth Analg 2003; 96:576-582.

46. Krishnan SK, Benzon HT, Siddiqui T, Canlas B. Pain on intramuscular injection of bupivacaine, ropivacaine, with and without dexamethasone. Reg Anesth Pain Med 2000; 25:615-619.

47. Plafki C, Steffen R, Willburger RE, Wittenberg $\mathrm{RH}$. Local anaesthetic injection with and without corticosteroids for subacromial impingement syndrome. Int Orthop 2000; 24:40-42.

48. Rasmussen SB, Saied NN, Bowens C Jr, Mercaldo ND, Schildcrout JS, Malchow RJ. Duration of upper and lower extremity peripheral nerve blockade is prolonged with dexamethasone when added to ropivacaine: A retrospective database analysis. Pain Med 2013; 14:1239-1247.
49. Golwala MP, Swadia VN, Aditi A, Dhimar, Sridbar NV. Pain relief by dexamethasone as an adjuvant to local anesthetics in supraclavicular brachial plexus block. J Anesth Clin Pharmacol 2009; 25:285-288.

50. Choi S, Rodseth R, McCartney CJ. Effects of dexamethasone as a local anaesthetic adjuvant for brachial plexus block: A systematic review and meta-analysis of randomized trials. $\mathrm{Br}$ J Anaesth 2014; 112:427-439.

51. Johansson A, Hao J, Sjölund B. Local corticosteroid application blocks transmission in normal nociceptive C-fibres. Acta Anaesthesiol Scand 1990; 34:335-338.

52. Attardi B, Takimoto K, Gealy R, Severns C, Levitan ES. Glucocorticoid induced up-regulation of a pituitary $\mathrm{K}+$ channel mRNA in vitro and in vivo. Receptors Channels 1993; 1:287-293.

53. Shishido $H$, Kikuchi $S$, Heckman $H$, Myers RR. Dexamethasone decreases blood flow in normal nerves and dorsal root ganglia. Spine (Phila Pa 1976) 2002; 27:581-586.

54. Conroy PH, Awad IT. Ultrasound-guided blocks for shoulder surgery. Curr Opin Anaesthesiol 2011; 24:638-643.

55. Neal JM, Gerancher JC, Hebl JR, Ilfeld BM, McCartney CJ, Franco CD, Hogan $\mathrm{QH}$. Upper extremity regional anesthesia: Essentials of our current understanding, 2008. Reg Anesth Pain Med 2009; 34:134-170.

56. Wang PH, Tsai CL, Lee JS, Wu KC, Cheng $\mathrm{KI}$, Jou IM. Effects of topical corticosteroids on the sciatic nerve: An experimental study to adduce the safety in treating carpal tunnel syndrome. J Hand Surg Eur 2011; 36:236-243.

57. Williams BA, Schott NJ, Mangione MP, Ibinson JW. Perineural dexamethasone and multimodal perineural analgesia: How much is too much? Anesth Analg 2014; 118:912-914.

58. Kawanishi R, Yamamoto K, Tobetto $Y$, Nomura K, Kato M, Go R, Tsutsumi YM, Tanaka K, Takeda Y. Perineural but not systemic low-dose dexamethasone prolongs the duration of interscalene block with ropivacaine: A prospective randomized trial. Local Reg Anesth 2014; 7:5-9.

59. Thomas S, Beevi S. Epidural dexamethasone reduces postoperative pain and analgesic requirements. Can J Anaesth 2006; 53:899-905.

6o. Sripada R, Bowens $C$ Jr. Regional anesthesia procedures for shoulder and upper arm surgery upper extremity update--2005 to present. Int Anesthesiol Clin 2012; 50:26-46. 